\title{
Pharmacist guidelines and considerations for treating $H$. pylori
}

\begin{abstract}
Helicobacter pylori (H. pylori) is considered one of the most major causes of gastrointestinal ulcers. With the ever expanding scope of pharmacists, providing appropriate recommendations to patients suffering from the disease remains a challenge. Considerations that must be taken into effect are resistance, patient health status, patient medications and compliance. Treatment options that are most common include a proton pump inhibitor with two different antibiotics, but recent cases of resistance is leading to different treatment approaches.
\end{abstract}

Keywords: helicobacter pylori, patients, proton pump inhibitor, antibiotics, pharmacists
Volume 7 Issue 2 - 2018

\author{
Ali Chaudhry \\ Shoppers Drug Mart, Thunder Bay, Canada
}

Correspondence: Ali Chaudhry, Shoppers Drug Mart, Thunder Bay, Ontario, Canada, Email ali_ch422@hotmail.com

Received: March 06, 2018 | Published: April 09, 2018

\section{Mini review}

Helicobacter pylori (H. pylori) infection remains one of the most common bacterial infections that affect humans, which can cause ulcers in the duodenum and the gastric lining of the stomach. ${ }^{1,2} H$. pylori is a gram negative bacteria which increases gastrin release from the gastric mucosa, resulting in the release of chemotactic cytokines, from an inflammatory response. ${ }^{2,3}$ Common symptoms include nausea, heartburn and indigestion. ${ }^{1,4}$ Eradication of this bacteria is not easy, and there are multiple factors which can affect eradication such as bacterial resistance, patient health status, patient's use of recreational drugs or alcohol, the patient's smoking status, patient medications or patient compliance. ${ }^{3,5}$

There are many methods available to diagnose $H$. pylori infections, such as serological tests, which measure IgG antibodies, which is effective approximately $80-95 \%$ of the time. ${ }^{1,2}$ Another diagnostic method used is the urea breathe test, in which a patient consumes ${ }^{13} \mathrm{C}$ or ${ }^{14} \mathrm{C}$, and the bacteria metabolises the urea quickly, resulting in the labelled carbon to be absorbed. The labelled carbon is then exhaled as carbon dioxide and measured. This method is highly effective, and with an effectiveness range of approximately 94-98\%. Another method to diagnose $H$. pylori is to perform an endoscopy, in which a biopsy of the stomach and the duodenum is performed. ${ }^{1,2}$ Once the biopsy is performed, H. pylori is diagnosed by either using the biopsy urease test, histological identification of the organisms, or the culture of the biopsy specimens. ${ }^{2}$

There are several treatment regimens available, first line treatment of H. pylori usually includes a Proton Pump Inhibitor (PPI), and two antibiotics, which is known as concomitant therapy. The combination of the two antibiotics can be either amoxicillin, 1gram, and clarithromycin, $500 \mathrm{mg}$, or metronidazole, $500 \mathrm{mg}$, and clarithromycin, $500 \mathrm{mg}$. These combinations are usually administered twice daily for approximately 14 days. ${ }^{1,2,6}$ These combinations are successful in achieving eradication rates of at least $80 \%{ }^{1,2,5}$ The most common PPIs are omeprazole $20 \mathrm{mg}$, pantoprazole $40 \mathrm{mg}$ and lansoprazole $30 \mathrm{mg}$, with the latter being the most common due to fact that it is considered to have the most potent inhibition against $H$. pylori urease activity, along with it being inexpensive and less common for drugdrug interactions. ${ }^{2}$
Each antibiotic has its own mechanism of action that helps eradicate the bacteria. ${ }^{4}$ Clarithromycin works by inhibits the protein synthesis of the bacteria ribosome. ${ }^{3,5}$ Metronidazole works by doing point mutations along certain genes, whereas amoxicillin works by inhibiting the cell wall synthesis. ${ }^{4}$ Where amoxicillin resistance rates are considered low, worldwide, clarithromycin and metronidazole have higher rates of resistance. ${ }^{3,6}$

Side effects from these combinations include diarrhea, taste disturbance, metallic taste, and nausea. ${ }^{2}$ Second line treatment for $H$. pylori usually, known as quadruple therapy, includes a PPI or a $\mathrm{H}_{2}$ receptor antagonist $\left(\mathrm{H}_{2} \mathrm{RA}\right)$, with bismuth, tetracycline, $500 \mathrm{mg}$, and metronidazole, $500 \mathrm{mg}^{2}{ }^{1,2,5}$ This regime is usually dosed four time daily for 14 days. ${ }^{2,5}$ This regime is usually successful in achieving an eradication rate of at least $75 \% .1$ Side effects from this regime usually include diarrhea, discolouration of the stools and tongue, metallic taste, and nausea. ${ }^{2}$

Other treatment options include, sequential and hybrid therapy. ${ }^{1}$ Sequential therapy involves the use of a PPI and amoxicillin for 5 days, followed by a PPI, clarithromycin and metronidazole for additional 5 days. ${ }^{1,2,4}$ Another treatment option, known as hybrid therapy, is the use of a PPI and amoxicillin for 7 days, which is then followed by a PPI, amoxicillin, clarithromycin and metronidazole for another 7 days. ${ }^{1,2}$ Both of these options are effective approximately $85-88 \%$ in eradication H. pylori. ${ }^{1}$

More recently, due to the increase in resistance, floroquinolones, more specifically, levofloxacin, is being used. ${ }^{1,4,6}$ Levofloxacin works by inhibiting bacterial topoisomerase II. ${ }^{5}$ It can be used in combination with amoxicillin 1 gram and a standard PPI, twice daily, for 10-14 days, which is known as levofloxacin triple. But levofloxacin only has to be dosed once daily, which can be confusing to some patients. . $^{1,5.6}$ This regime has an eradication rate of approximately $74-85 \%{ }^{3}$ Levofloxacin can also be used with what is considered levofloxacin sequential therapy, in which the dosing regime starts as amoxicillin 1 gram and a PPI for 5-7days, then amoxicillin, PPI, levofloxacin $500 \mathrm{mg}$ daily and nitroimidazole $500 \mathrm{mg}$, twice daily, for 5-7 days. ${ }^{1,3,6}$ This therapy has an eradication rate of about $87.8 \% .^{1,6}$ Levofloxacin can also be dosed at $250 \mathrm{mg}$ daily, with a PPI double dose once daily, doxycycline $100 \mathrm{mg}$ daily and nitazoxanide 500mg twice daily for 
7-10 days, which is known LOAD therapy.,3 This therapy regime has an eradication rate of approximately $90 \%{ }^{1}$

When choosing an antibiotic combination, one of the factors that must be considered is the patient's allergy status, meaning if they are allergic to amoxicillin, then metronidazole should be given. ${ }^{1-3}$ Another factor is the patient's willingness to not drink alcohol for the duration of treatment, if the patient is unwilling to stop alcohol while on treatment, it would advisable to use amoxicillin over metronidazole. When considering the patient's other medications, it would important to note that clarithromycin and omeprazole are metabolised by cytochrome-P450 $\left(\mathrm{CYP}_{450}\right)$ enzymes, which will affect the effectiveness and safety of the patient's medication and the antibiotic. Clarithromycin will interact with digoxin, atorvastatin, warfarin, diazepam, etc. while levofloxacin will interaction with warfarin, calcium and iron supplements, hydroxychloroquine, escitalopram, etc. ${ }^{1,2,4}$ If drug-drug interactions are of concern, and the patient cannot stop the chronic medication for the duration of therapy, then it would be advisable to use second line treatment. Levofloxacin is commonly used if first and second line treatments fail. ${ }^{4}$ If compliance may be an issue, then it recommended that a HP-PAC be used, which provided blister packed cards, with the doses for morning and evening clearly identified. $^{2}$

Considering all therapies, it should be noted that concomitant, hybrid and sequential therapy are all involve the same medications, and are similar in effectiveness, whereas therapy quadruple therapy is considered effective, but less than the other three therapies mentioned.
However it should be noted that none of the therapies mentioned are $100 \%$ effective in eradication $H$. pylori and with increasing resistance rates, new therapy options may be required. ${ }^{1,2,6}$

\section{Acknowledgements}

None.

\section{Conflict of interest}

The author declares that there is no conflict of interest.

\section{References}

1. Chey WD, Leontiadis GI, Howden CW, et al. ACG Clinical Guideline: Treatment of Helicobacter pylori Infection. Am $J$ Gastroenterol. 2017;112(2):212-238

2. http://www.rxfiles.ca/rxfiles/uploads/documents/hpylori.pdf

3. Kim Su Young, Choi Duck Joo, Chung Jun-Won. Antibiotic treatment for Helicobacter pylori : Is the end coming?. World J Gastrointest Pharmacol Ther. 2015;6(4):183-198.

4. Safavi Maliheh, Sabourian Reyhaneh, Foroumadi Alireza. Treatment of Helicobacter pylori infection: Current and future insights. World J Clin Cases. 2016;4(1):5-19.

5. https://www.cdc.gov/ulcer/files/hpfacts.pdf

6. Fallone Carlo A, Chiba Naoki, Veldhuyzen van Zanten Sander, et al. The Toronto Consensus for the Treatment of Helicobacter pylori Infection in Adults. Gastroenterology. 2016;151(1):51-69. 